\title{
Parcellation of Sensorimotor Transformations for Arm Movements
}

\author{
Martha Flanders and John F. Soechting \\ Department of Physiology, University of Minnesota, Minneapolis, Minnesota 55455
}

Pointing to a visual target in 3-dimensional space requires a neural transformation from a visually derived representation of target location to an appropriate pattern of activity in arm muscles. Previous results suggested that 1 step in this process involves a transformation from a representation of target location to a representation of intended arm orientation, and that the neural implementation of this transformation involves a linear approximation to the mathematically exact, nonlinear solution. These results led to the hypothesis that the transformation is parceled into 2 separate channels. In 1 channel a representation of target azimuth is transformed into a representation of arm yaw angles, while in the other channel representations of target distance and target elevation are transformed into a representation of arm elevation angles. The present experiments tested this hypothesis by measuring the errors made by human subjects as they pointed to various parameters of the remembered location of a target in space. The results show that subjects can use the 2 hypothesized channels separately. For example, subjects can accurately point to the target's azimuth while ignoring the target's elevation and distance. The results also show that subjects are unable to point to the target's elevation while ignoring the target's distance, consistent with the hypothesis that information about target elevation and target distance is tied together in the same channel. The parcellation demonstrated in this study is compared to reports of parceled sensorimotor transformation in other vertebrate species.

Although arm movements are ultimately accomplished by the production of muscular force, the nervous system "plans" these movements in terms of kinematics. By "planning" we mean that prior to the activation of motor units, groups of cells within central nervous system exhibit activity related to kinematic parameters such as position and direction. For example, for an arm movement to a visible target, the target position is initially represented by the activity of retinal neurons. A visually derived representation of target position is then thought to be combined with a kinesthetically derived representation of initial arm position to form a representation of the direction and amplitude of the intended movement (Soechting and Flanders, 1990). In

\footnotetext{
Received Feb. 1, 1990; accepted April 2, 1990.

This work was supported by National Institute of Neurological Disorders and Stroke Grants NS-27484 and NS-15018.

Correspondence should be addressed to Dr. Martha Flanders, Department of Physiology, 6-255 Millard Hall, University of Minnesota, Minneapolis, MN 55455. Copyright $\odot 1990$ Society for Neuroscience $0270-6474 / 90 / 072420-08 \$ 03.00 / 0$
}

fact, the activity of neurons in the motor cortex is correlated with the direction of the movement that is needed to get from the initial position to the target position (Georgopoulos, 1986; Georgopoulos et al., 1988).

In this paper we present a series of experiments designed to test an hypothesis concerning the sensorimotor transformation from a representation of target position to a representation of the intended movement (Fig. 1). Our hypothesis is based on the results of previous expcriments, in which we askcd human subjects to point to the remembered location of a target in 3-dimensional space (Soechting and Flanders, 1989a). Subjects made large errors in pointing to the virtual targets, and from these errors we deduced the stages and algorithms involved in this sensorimotor transformation. Our results suggested that a retinocentric representation of target position is ultimately transformed into a neural representation of the distance and direction of the target from the subject's shoulder (Soechting et al., 1990). This shoulder-centered representation of target position is then transformed into a representation of the intended, final arm orientations for the movement. The parameters that represent the location of the target relative to the shoulder are the distance, the elevation (up/down angle), and the azimuth (right/left angle). The parameters that represent upper arm and forearm orientations are elevation (angle relative to the vertical) and yaw (angle relative to the anterior direction) (Soechting and Ross, 1984). Our data suggested that the transformation to intended arm orientations involves a linear approximation to the mathematically exact solution (Soechting and Flanders, 1989b). We explained the observed errors in pointing by showing that the final arm elevation is a linear function of target distance and target elevation, and the final arm yaw is a linear function of target azimuth. If this interpretation is correct, it implies that information about distance and elevation is processed in a channel that is separate and distinct from the channel that processes information about azimuth.

Thus, we hypothesize that there is a "parcellation" in the transformation between a shoulder-centered representation of target position and a representation of intended arm orientations (cf. Grobstein, 1988). As diagrammed in Figure 1, the neural representation of these parameters is parceled into 2 distinct transformations. If this hypothesis is correct, subjects should be able to make a movement that matches only the azimuth of a virtual target, without using information about the target's elevation and distance. Similarly, subjects should be able to match target elevation and distance, without matching target azimuth. However, since we hypothesize that distance and elevation information are processed together in the same channel, we predict that subjects should not be able to match target elevation alone, without moving to the target's distance. 


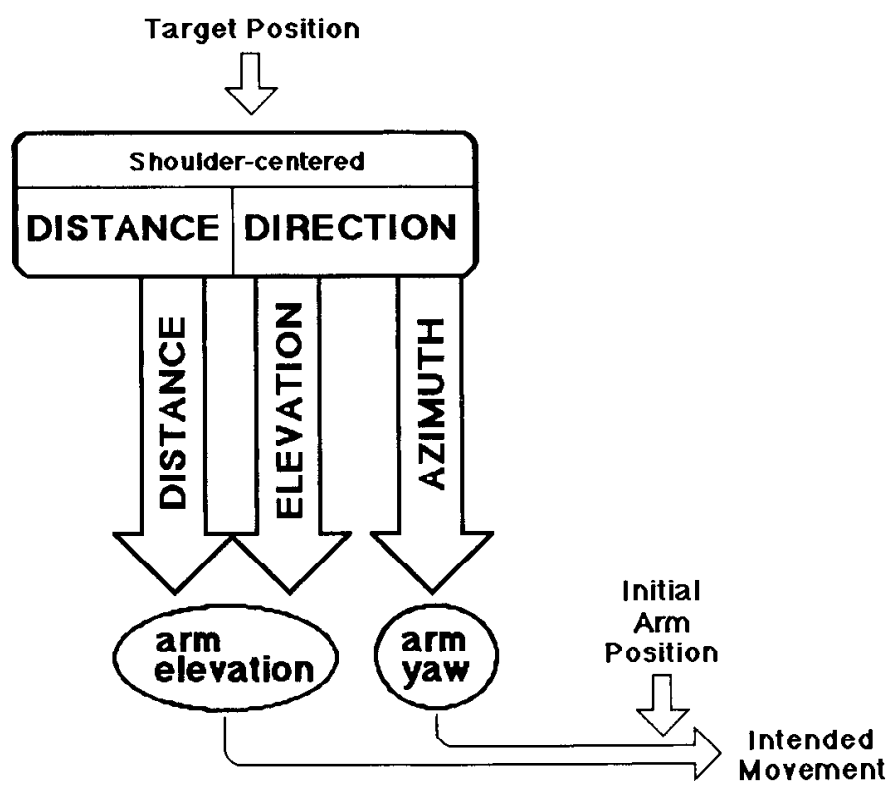

Figure 1. Parcellation hypothesis. A neural representation of target location is ultimately transformed into a representation of the distance and direction of the target from the shoulder. Information about target distance and direction are used together in the transformation to a neural representation of the final arm elevation angles of the intended movement. Information about target azimuth is transformed into a representation of arm yaw angles in a separate channel. The representations of the intended, final arm orientations are combined with a neural representation of the initial arm position to form a representation of the distance and direction of the intended arm movement.

\section{Materials and Methods}

\section{Motor tasks}

The motor tasks used in these experiments were similar to those we have described previously (Soechting and Flanders, 1989a). Subjects stood erect. They were presented with targets at locations that encompassed most of the range of arm motion. The target was the tip of a stylus held by the experimenter. The subjects were asked to remember the location of the target, the target was removed from the subjects' view, and the subjects produced a pointing movement with their right arm. The movement was made either in the dark or with the room lights on. The specific instructions for each experimental condition will be described in more detail below. Each experimental condition consisted of between 60 and 100 target locations, varied at random.
There were 6 different experimental conditions. At least 4 subjects were tested in each condition.

Virtual target (dark). This was the control condition, used to assess the subjects' performance in the other experimental conditions. The subjects were asked to point to the remembered target location with their index finger in the dark. The results of this experiment, for 4 of the 8 subjects, have been described in detail previously (Soechting and Flanders, 1989a).

Match elevation and distance (dark). This task is illustrated in Figure $2 A$. Subjects were asked to restrict their arm movements to the sagittal plane passing through the shoulder and to point to a location in this plane that was at the same distance $(R)$ and elevation $(\Psi)$ as the target. Thus they were asked to ignore the target's azimuth. According to our hypothesis, subjects should be able to perform this task. The yaw angles of the arm should always be close to zero, and the elevation angles $(\theta-$ upper arm; $\beta$-forearm) should be related to a linear combination of distance and elevation of the target, the same as if the subjects had pointed to the actual target (Soechting and Flanders, 1989b)

Match elevation and distance (light). The only difference between this task and the preceding one was that the room lights were not extinguished. In the control condition, vision of the arm leads to only minor differences in performance (Soechting and Flanders, 1989a; Soechting et al. 1990). Therefore, one would predict that vision of the arm, or its occlusion, should not affect performance when subjects are asked to match only target elevation and distance.

Match elevation (light). In this task, subjects were asked to ignore target distance. They were also asked to ignore target azimuth by moving their arm in the sagittal plane. They were to place their fingertip somewhere on the line between the shoulder and the target's virtual location, rotated into the sagittal plane of the shoulder. According to our hypothesis, the error in the subjects' performance should be appreciably larger in this experimental condition than in the 3 preceding conditions.

Match elevation (pointer). Subjects were given a pointer which was about a meter long. They were asked to hold it at shoulder level such that it pivoted about an axis close to the center of rotation of the shoulder joint. They were instructed to match the elevation of the pointer to the target's elevation by rotation of the pointer in the sagittal plane. This task required subjects to make wrist and hand movements, in contrast to the movements at the more proximal joints required by the previous tasks. If the difficulty in the preceding task stemmed from a linkage of distance and elevation parameters in the transformation to arm orientation, subjects should be able to match target elevation with the pointer much better than they are able to with their arm. Alternatively, if the difficulty in the preceding task stemmed from an inability to perceive target elevation, subjects would also do poorly with the pointer. The room lights were on in this experimental condition.

Match azimuth (dark). In this task, subjects were asked to match the azimuth $(\chi)$ of the target location, ignoring the target's distance and elevation. As indicated schematically in Figure $2 B$, they werc instructcd to keep their upper arm close to vertical and their forearm close to horizontal. They were asked to move their arm so that the fingertip was in the vertical plane passing through the shoulder and the target, that is, at the target's azimuth. These experiments were performed in the dark.
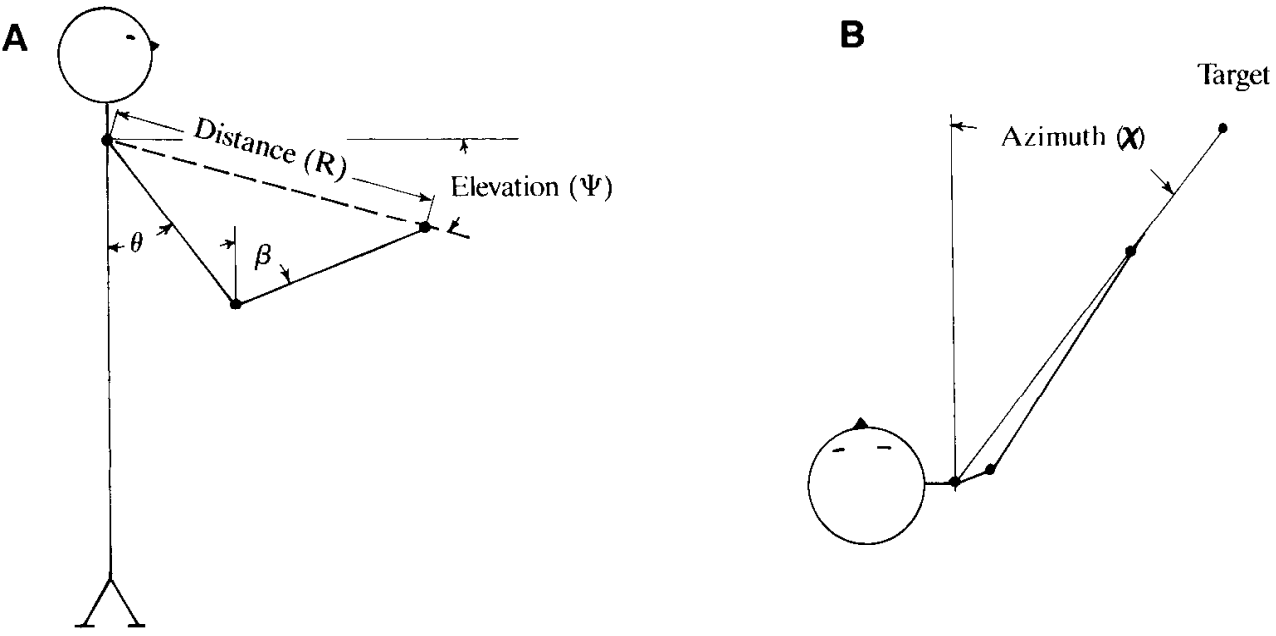

Figure 2. Subjects were asked to make arm movements that matched some of the target parameters, while ignoring others. In $A$, a schematic drawing indicates that the subject is matching the distance and elevation of the target, while ignoring the target azimuth by confining the arm movement to the sagittal plane. In $B$, the subject is matching the target's azimuth, while ignoring distance and elevation by keeping the upper arm close to vertical and moving the forearm in the horizontal plane. 


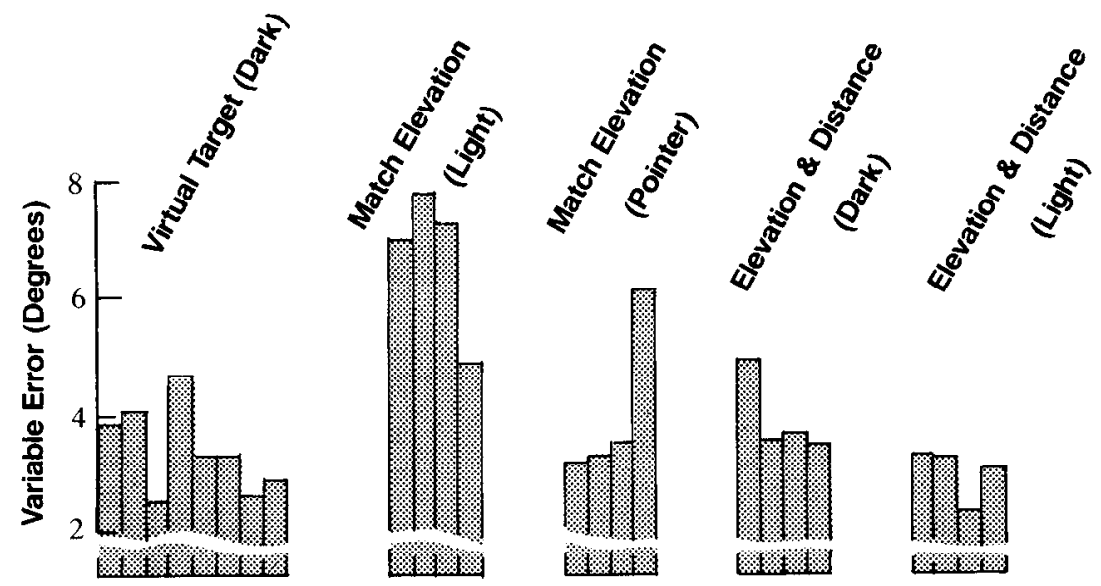

Figure 3. Finger elevation: performance in matching target elevation under various experimental conditions (see Materials and Methods). Variable error was largest when subjects attempted to match elevation while ignoring target distance.

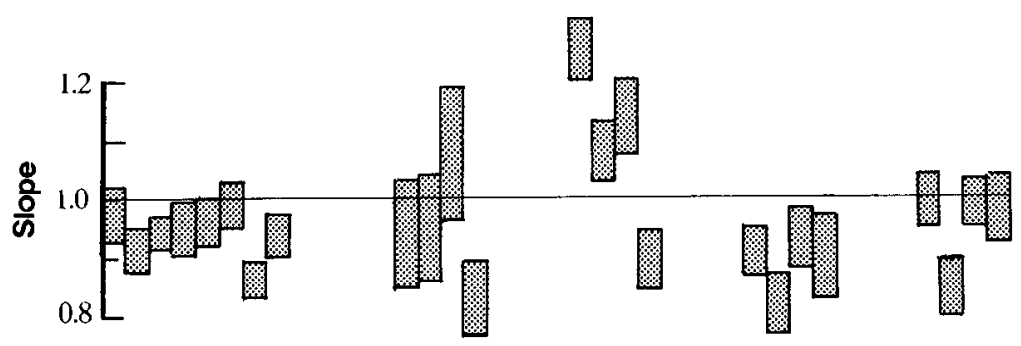

For each of the experimental conditions, the requirements of the task were demonstrated to each subject and he or she was given several practice trials to make certain that the instructions were well understood.

\section{Recording system and data analysis}

The location of the target was measured ultrasonically with a resolution of $0.1 \mathrm{~mm}$. The ultrasound system detected the 3-dimensional position of a spark gap at the tip of the stylus, which constituted the target. To measure arm orientations, additional styli were attached to the subject's arm at the shoulder, elbow, and wrist. Finger position was measured by means of a fourth stylus held by the subject.

We used a multivariate linear regression analysis to determine the manner in which each of the 3 parameters defining finger position (distance, elevation, and azimuth) depended on all 3 parameters defining target position. We constructed a polynomial fit to model finger position as a function of target parameters. In our models, we sometimes used only linear terms, and sometimes used quadratic and cubic polynomial terms as well. In both cases, however, only those terms with coefficients different from zero at the $95 \%$ confidence level were retained in the model.

We assessed the subjects' performance according to 2 criteria: accuracy and variability. For accuracy we used the linear model. For accurate performance, the slope of the regression between target and finger position parameters should be unity (for example, target elevation should be equal to finger elevation). For variability we used the model containing up to cubic terms (the "cubic model"). We define variable error as the square root of the variance of the difference between the actual finger position and the position predicted by the model.

We performed a similar regression analysis to determine the manner in which parameters defining arm orientation depended on the parameters defining target location. We assessed the linearity of this relationship by calculating the extent to which the cubic polynomial gave a better fit to the data than did the linear model: we will report the square root of the difference in the variances of these 2 models.

More information about the recording system and data analysis can be found in previous publications (Soechting and Flanders, 1989a, b).

\section{Results}

We will present our results in 2 sections. First, we will report the overall performance under the various experimental con- ditions. We will report the errors in finger position in terms of elevation, distance, and azimuth. Next, we will examine the transformations between target parameters and arm orientations and the extent to which these transformations differ under various cxperimental conditions.

\section{Performance under various conditions}

Finger elevation. Figure 3 illustrates the principal result of this study. As is predicted by our hypothesis, the subjects' variability in performance was the greatest when they attempted to match only the target's elevation, ignoring distance. In Figure 3, we summarize the performance in terms of elevation for 5 different experimental conditions. The bars in the top row indicate the variable error in elevation, measured in degrees, for each subject. Variable error was defined to be the error that could not be accounted for by the regression model, which could include up to cubic polynomial terms. Of the 20 possible terms in this regression, generally about 4-6 terms had coefficients that were significant. Therefore, the variable error, as we have defined it, provides a measure of the uncertainty in the subject's performance.

In the control condition, in which subjects pointed to a virtual target in the dark, the variable error in elevation was $3.40^{\circ} \pm$ $0.70^{\circ}$ (mean and standard error for 8 subjects). When the subjects' movement was restricted to the sagittal plane and they attempted to match only elevation and distance (ignoring azimuth), the variable error in elevation was comparable: $3.89^{\circ} \pm$ $0.60^{\circ}$ in the dark and $3.03^{\circ} \pm 0.31^{\circ}$ in the light (Fig. 3, top right). However, when subjects attempted to match only the elevation of the target in the light (ignoring distance and azimuth), the variable error in elevation $\left(6.70^{\circ} \pm 1.10^{\circ}\right.$ ) was about twice as large as the error in matching elevation in the other conditions (Fig. 3, top center). Thus, there was a $100 \%$ increase in variable error under this experimental condition. 


\section{Virtual Target}

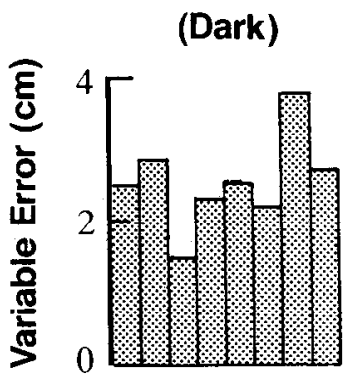

Match Distance \& Elevation

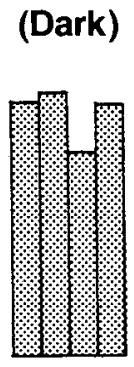

(Light)

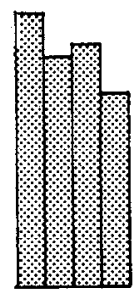

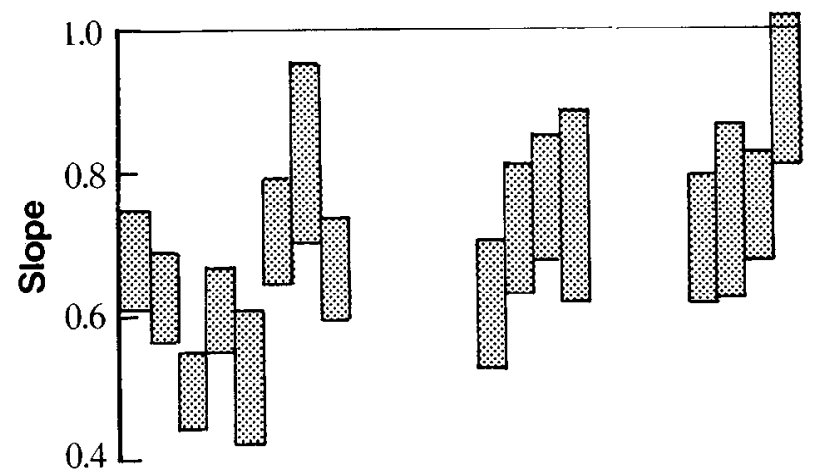

Figure 4. Finger distance: performance in matching target distance. Performance in 2 experimental conditions where subjects were asked to ignore target azimuth was comparable to performance in the control condition.

The subjects' uncertainty in matching only elevation was diminished substantially when they were permitted to use a pointer for this task (Fig. 3, top center). Under this condition the variable error was $4.00^{\circ} \pm 1.20^{\circ}$, and in only 1 of the 4 subjects did this error exceed the average for the control condition by an appreciable amount.

The principal difference between the 2 experimental conditions in which only elevation was to be matched was that the former involved primarily motion at the shoulder and elbow and the latter primarily motion at the wrist and hand. Therefore, the subjects' greater difficulty with the arm movement (compared to the pointer movement) is due to the fact that a transformation to shoulder and elbow orientations is not required in the latter task. It cannot be attributed to perceptual difficulties in deriving the elevation of the target independently of other target parameters. In other words, as implied by the hypothesis illustrated in Figure 1, the difficulty arises because subjects were attempting to derive arm elevations utilizing only target elevation and ignoring target distance.

This conclusion is reinforced by a finding we have reported previously (Soechting et al., 1990). When subjects were asked to move their finger to a point halfway from the shoulder in the dark, the variable error in elevation $\left(3.50^{\circ} \pm 0.24^{\circ}\right)$ was comparable to the error under control conditions. An increase in variability in performance arises only when subjects are instructed to ignore distance information, but not when they are instructed to alter it.

The lower row in Figure 3 provides an assessment of the
Virtual Target (Dark)
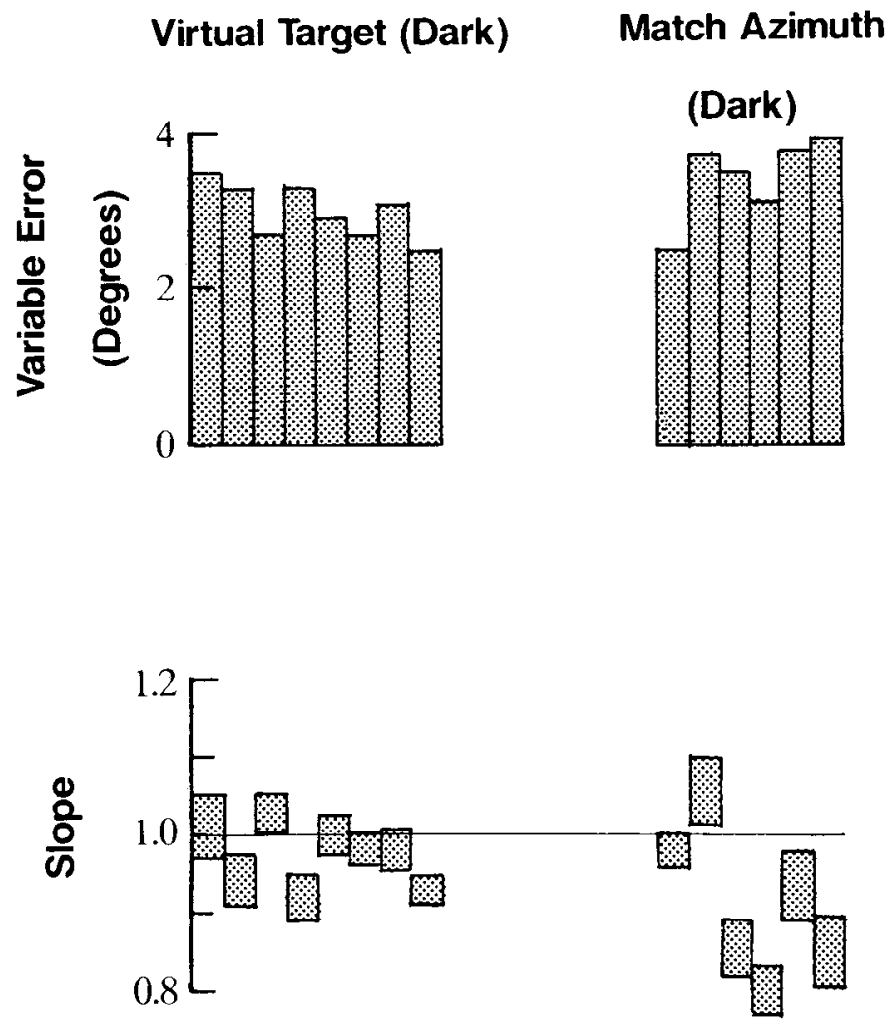

Figure 5. Finger azimuth: performance in matching target azimuth. When subjects ignored target elevation and distance, their performance was comparable to performance under control conditions.

subjects' accuracy in matching elevation in the various experimental conditions. Each bar indicates the $95 \%$ confidence interval of the slope of the linear regression between target elevation and finger elevation. If, on average, subjects had matched target elevation perfectly, the slope would be 1.0. In the control condition, the slope was in fact slightly less than this value: 0.94 \pm 0.04 . In the 4 matching conditions, the intersubject variability in the estimates of the slope tended to increase, especially for the 2 conditions in which subjects matched target elevations only. However, the values obtained in the 4 matching conditions did not differ substantially from control values $(0.89 \pm 0.05$ for elevation and distance in the dark, $0.95 \pm 0.07$ in the light; 0.95 \pm 0.09 for elevation only with the arm, $1.09 \pm 0.13$ with the pointer).

Finger distance. According to our hypothesis, the variable error in distance when subjects match distance and elevation together should be comparable to the variable error in distance when subjects point to a virtual target in the dark. Figure 4 shows how well this prediction is satisfied by the experimental results. In the control condition, Virtual Target (Dark), the variable error in distance averaged $2.53 \pm 0.61 \mathrm{~cm}$. The error was only about $30 \%$ larger when subjects matched distance and elevation in the dark $(3.40 \pm 0.34 \mathrm{~cm})$ or in the light $(3.28 \pm$ $0.41 \mathrm{~cm})$. The slopes of the regression between target distance and finger distance (lower row in Fig. 4) were consistently less than 1.0: in all 3 experimental conditions, subjects tended to undershoot the target. The values were comparable for all 3 conditions (virtual target: $0.64 \pm 0.11$; match elevation and distance, dark: $0.71 \pm 0.07$, light: $0.78 \pm 0.09$ ).

Finger azimuth. Subjects were able to match the azimuth of 


\begin{tabular}{|c|c|c|c|c|c|c|c|c|c|}
\hline & \multicolumn{4}{|c|}{ Linear model } & \multicolumn{3}{|c|}{ Variable error } & \multicolumn{2}{|l|}{$r^{2}$} \\
\hline & $\mathrm{DC}$ & $\mathrm{R}$ & $x$ & $\Psi$ & Linear & Cubic & $\Delta$ & Linear & Cubic \\
\hline $\begin{array}{l}\text { Virtual target } \\
\text { (dark) }\end{array}$ & $\begin{array}{l}-8.3 \\
(12.3)\end{array}$ & $\begin{array}{c}1.13 \\
(0.19)\end{array}$ & 0 & $\begin{array}{c}0.93 \\
(0.07)\end{array}$ & $\begin{array}{l}5.74 \\
(0.89)\end{array}$ & $\begin{array}{l}5.28 \\
(0.94)\end{array}$ & $\begin{array}{c}1.96 \\
(0.93)\end{array}$ & 0.944 & 0.951 \\
\hline $\begin{array}{l}\text { Elevation only } \\
\text { (light) }\end{array}$ & $\begin{array}{c}17.0 \\
(16.4)\end{array}$ & $\begin{array}{c}0.19 \\
(0.19)\end{array}$ & $\begin{array}{c}0.05 \\
(0.09)\end{array}$ & $\begin{array}{c}0.69 \\
(0.19)\end{array}$ & $\begin{array}{l}9.74 \\
(2.71)\end{array}$ & $\begin{array}{l}9.54 \\
(2.68)\end{array}$ & $\begin{array}{c}1.39 \\
(1.39)\end{array}$ & 0.744 & 0.751 \\
\hline $\begin{array}{l}\text { Elevation and } \\
\text { distance (dark) }\end{array}$ & $\begin{array}{r}-24.1 \\
(9.6)\end{array}$ & $\begin{array}{c}1.12 \\
(0.14)\end{array}$ & $\begin{array}{c}0.16 \\
(0.03)\end{array}$ & $\begin{array}{c}0.80 \\
(0.09)\end{array}$ & $\begin{array}{l}7.39 \\
(1.01)\end{array}$ & $\begin{array}{c}6.00 \\
(0.66)\end{array}$ & $\begin{array}{c}4.23 \\
(1.16)\end{array}$ & 0.882 & 0.920 \\
\hline $\begin{array}{l}\text { Elevation and } \\
\text { distance (light) }\end{array}$ & $\begin{array}{r}-33.3 \\
(13.5)\end{array}$ & $\begin{array}{c}1.32 \\
(0.18)\end{array}$ & $\begin{array}{l}0.16 \\
(0.06)\end{array}$ & $\begin{array}{c}0.87 \\
(0.06)\end{array}$ & $\begin{array}{c}6.71 \\
(0.83)\end{array}$ & $\begin{array}{c}5.70 \\
(0.94)\end{array}$ & $\begin{array}{l}3.25 \\
(1.35)\end{array}$ & 0.903 & 0.932 \\
\hline
\end{tabular}

the target, ignoring elevation and distance. The performance was almost as good as when they pointed to the virtual target itself. In Figure 5, we compare the results of these 2 experimental conditions. Variable error in azimuth was only slightly larger when subjects attempted to match azimuth alone $\left(3.45^{\circ} \pm 0.42^{\circ}\right.$ vs $3.01^{\circ} \pm 0.35^{\circ}$ ). As was the case for elevation, there was greater intersubject variability in the estimated values of the slopes of the regression between target and finger azimuth $(0.91 \pm 0.09$ vs $0.97 \pm 0.04)$.

\section{Relationships between arm orientations and target parameters}

The data in Figures 3-5 are consistent with the hypothesis that the transformation from target parameters to arm orientations is parceled into 2 components: 1 involving target distance and elevation to derive arm elevation angles, and the other involving only target azimuth to derive arm yaw angles. When subjects point to a virtual target, the arm orientations are highly correlated with a linear combination of target parameters (Soechting and Flanders, 1989b). For motor tasks which involve only 1 or the other of the 2 hypothesized channels, we would expect to find that arm orientations are correlated with the same linear combination of target parameters as when subjects pointed to a virtual target.

Upper arm and forearm elevations. The results summarized in Tables 1 and 2 indicate the extent to which this prediction was satisfied for the upper arm elevation $-\theta$ (Table 1) and for forearm elevation $-\beta$ (Table 2 ). In these tables we present the data for the 3 experimental conditions in which subjects matched either target elevation alone or a combination of target elevation and distance by means of arm movements in the sagittal plane.
For each experimental condition, we calculated the average of the regression coefficients of the linear model (including an offset or "DC" term), using only those that were statistically significant for all the subjects. Tables 1 and 2 also list the variable error of the linear model and the variable error of the cubic models. The amount by which the cubic model improved the fit of the data is listed under $\Delta$ (see Materials and Methods).

In the control condition, when subjects point to a virtual target in the dark, $\theta$ is related about equally to target distance $(R)$ measured in $\mathrm{cm}$ and target elevation $(\Psi)$ measured in degrees (top line of Table 1). For both target distance and target elevation, the regression coefficient is positive and close to unity. In contrast, $\beta$ is negatively correlated with target elevation, but the absolute value of the regression coefficient is still close to unity (top line of Table 2). The dependence of $\beta$ on distance is about half that of $\theta$. Neither $\theta$ nor $\beta$ is significantly related to target azimuth $(\chi)$. The nonlinear model gave only a modest improvement in fit $(\Delta)$. The $\Delta$ values of 1.96 (for $\theta$ ) and 3.02 (for $\beta$ ) are much smaller than the previously reported values of 8.51 and 5.50 (respectively) for movements that do not involve linear approximations (Soechting and Flanders, 1989b).

The results were similar when subjects matched only elevation and distance, either in the dark or in the light (bottom rows in Tables 1 and 2). The values of the regression coefficients on $R$ and $\Psi$ were about the same as those found when subjects pointed directly to the target. The variable error for $\theta$ increased slightly, while that for $\beta$ actually decreased, and the cubic model gave only a modest improvement. The major difference is that now both $\theta$ and $\beta$ depend to a small but statistically significant amount on target azimuth $(\chi)$. Both arm elevations were larger for targets

\begin{tabular}{|c|c|c|c|c|c|c|c|c|c|}
\hline & \multicolumn{4}{|c|}{ Linear model } & \multicolumn{3}{|c|}{ Variable error } & \multicolumn{2}{|l|}{$r^{2}$} \\
\hline & $\overline{\mathrm{DC}}$ & $\mathrm{R}$ & $x$ & $\Psi$ & Linear & Cubic & $\Delta$ & Linear & Cubic \\
\hline $\begin{array}{l}\text { Virtual target } \\
\text { (dark) }\end{array}$ & $\begin{array}{l}46.6 \\
(15.4)\end{array}$ & $\begin{array}{c}0.51 \\
(0.21)\end{array}$ & 0 & $\begin{array}{c}-0.95 \\
(0.07)\end{array}$ & $\begin{array}{l}7.01 \\
(1.17)\end{array}$ & $\begin{array}{c}6.24 \\
(1.36)\end{array}$ & $\begin{array}{c}3.02 \\
(0.75)\end{array}$ & 0.890 & 0.911 \\
\hline $\begin{array}{l}\text { Elevation only } \\
\text { (light) }\end{array}$ & $\begin{array}{c}59.0 \\
(16.1)\end{array}$ & $\begin{array}{c}0.14 \\
(0.08)\end{array}$ & 0 & $\begin{array}{r}-0.97 \\
(0.14)\end{array}$ & $\begin{array}{c}8.51 \\
(2.56)\end{array}$ & $\begin{array}{c}7.59 \\
(2.47)\end{array}$ & $\begin{array}{l}3.17 \\
(2.26)\end{array}$ & 0.888 & 0.909 \\
\hline $\begin{array}{l}\text { Elevation and } \\
\text { distance (dark) }\end{array}$ & $\begin{array}{c}50.4 \\
(14.2)\end{array}$ & $\begin{array}{c}0.36 \\
(0.13)\end{array}$ & $\begin{array}{l}0.10 \\
(0.04)\end{array}$ & $\begin{array}{c}-0.87 \\
(0.07)\end{array}$ & $\begin{array}{c}5.87 \\
(1.17)\end{array}$ & $\begin{array}{c}5.13 \\
(0.96)\end{array}$ & $\begin{array}{c}2.77 \\
(0.99)\end{array}$ & 0.922 & 0.940 \\
\hline $\begin{array}{l}\text { Elevation and } \\
\text { distance (light) }\end{array}$ & $\begin{array}{c}50.6 \\
(14.7)\end{array}$ & $\begin{array}{c}0.40 \\
(0.12)\end{array}$ & $\begin{array}{c}0.10 \\
(0.10)\end{array}$ & $\begin{array}{c}-0.96 \\
(0.09)\end{array}$ & $\begin{array}{l}5.48 \\
(1.26)\end{array}$ & $\begin{array}{c}4.65 \\
(1.56)\end{array}$ & $\begin{array}{c}2.72 \\
(0.41)\end{array}$ & 0.945 & 0.961 \\
\hline
\end{tabular}


Table 3. Forearm yaw $-\alpha$

\begin{tabular}{|c|c|c|c|c|c|c|c|c|c|}
\hline & \multicolumn{4}{|c|}{ Linear model } & \multicolumn{3}{|c|}{ Variable error } & \multicolumn{2}{|l|}{$r^{2}$} \\
\hline & $\overline{\mathrm{DC}}$ & $\mathbf{R}$ & $x$ & $\Psi$ & Linear & Cubic & $\Delta$ & Linear & Cubic \\
\hline $\begin{array}{l}\text { Virtual target } \\
\text { (dark) }\end{array}$ & $\begin{array}{r}-12.6 \\
(12.2)\end{array}$ & $\begin{array}{l}0.05 \\
(0.16)\end{array}$ & $\begin{array}{c}1.03 \\
(0.08)\end{array}$ & $\begin{array}{c}-0.24 \\
(0.28)\end{array}$ & $\begin{array}{l}6.86 \\
(2.37)\end{array}$ & $\begin{array}{c}5.88 \\
(1.57)\end{array}$ & $\begin{array}{c}3.29 \\
(2.21)\end{array}$ & 0.947 & 0.962 \\
\hline $\begin{array}{l}\text { Azimuth only } \\
\text { (dark) }\end{array}$ & $\begin{array}{l}-5.2 \\
(5.6)\end{array}$ & $\begin{array}{c}0.01 \\
(0.06)\end{array}$ & $\begin{array}{c}0.82 \\
(0.14)\end{array}$ & $\begin{array}{c}0.04 \\
(0.09)\end{array}$ & $\begin{array}{l}4.19 \\
(0.84)\end{array}$ & $\begin{array}{c}4.04 \\
(0.71)\end{array}$ & $\begin{array}{c}0.82 \\
(0.85)\end{array}$ & 0.961 & 0.964 \\
\hline
\end{tabular}

located laterally than for targets located medially (but at the same distance and elevation). This corresponded to a small positive regression coefficient for target azimuth.

One possibility that could account for this positive correlation of $\theta$ and $\beta$ with target azimuth is that subjects used an origin to define distance and direction which was medial to the shoulder (Soechting et al., 1990), whereas in our calculations, we used an origin at the shoulder. If we instead used an origin at the center of the head, the value of the regression coefficient on azimuth would change sign for these experimental conditions. For example, in matching elevation and distance in the dark, the regression of $\theta$ and $\chi$ gave +0.16 when target location was measured from the shoulder and -0.18 when it was measured from the head. Similarly, the results for $\beta$ were +0.10 and -0.11 , respectively. While we did not pursue this issue further, these results indicate that had we measured distance and direction from a point about halfway between the center of the head and the shoulder, arm elevation would have been statistically independent of target azimuth. Experiments designed to identify the origin of the coordinate system used by subjects for pointing movements of the arm indicated that this origin was medial to the shoulder by about $40 \%$ of the distance from the shoulder to the center of the head (Soechting et al., 1990).

The relationship between arm elevation and target parameters was different when subjects attempted to match only target elevation. In accord with the instruction, the dependence of $\theta$ and $\beta$ on distance $(R)$ was substantially reduced (by $83 \%$ for $\theta$ and by $73 \%$ for $\beta$ ). Table 2 shows that the dependence of $\beta$ on target elevation $(\Psi)$ was about the same as in the control condition, as was the size of the variable error and the correlation coefficient $\left(r^{2}\right)$. This was not the case for $\theta$ (Table 1). For $\theta$, the slope of the regression on target elevation $(\Psi)$ decreased by $25 \%$ compared to control, the variable error increased by $70 \%$, and the correlation coefficient was substantially lower. Apparently, the major source of variability in finger location in this experimental condition (Fig. 3) was the increased variability in upper arm elevation.

Forearm azimuth. When subjects attempted to match azimuth only, the upper arm was close to vertical. Therefore, only forearm yaw was correlated with target azimuth. The data in Table 3 show that the variable error for forearm yaw was actually less when subjects matched only the azimuth of the target than when they pointed directly to the virtual target (4.19 for azimuth only compared to 6.86 for the control condition). The relationship between yaw and azimuth was very linear during matching; the cubic model decreased the variable error only slightly $(\Delta=$ 0.82 ). In both experimental conditions, forearm yaw depended only to a minor degree on target distance and elevation. The slope of the regression on target azimuth $(\chi)$ was slightly less when subjects matched only the azimuth of the target.

\section{Discussion}

The results presented above support the hypothesis that a transformation from the neural representation of target position to the neural representation of arm orientation is parceled into 2 distinct channels (Fig. 1). Our previous results suggested that the neural representation of intended arm elevation is derived from a linear combination of target elevation and distance, and arm yaw is a linear function of target azimuth. We have now demonstrated that these computations can be performed independently of one another.

We have demonstrated that (1) subjects are able to use these 2 channels separately and (2) when they do so they use a linear transformation that is similar to the one used during normal movements to virtual targets. We demonstrated this by asking subjects to ignore certain target parameters. When subjects ignored target azimuth, the transformation to arm elevations was similar to that observed under control conditions (Figs. 3, 4; Tables 1, 2). When they ignored elevation and distance, the transformation to forearm yaw was low in variable error (Fig. 5) and linear (Table 3 ).

We also demonstrated that, in accord with the hypothesis, subjects are unable to precisely compute arm elevations without using information about target distance. When subjects were asked to ignore target distance, the variable error in finger position increased by approximately $100 \%$ over control conditions. We believe that this finding provides the most critical support for the hypothesis. A performance that is not degraded (as in the other experimental conditions) could conceivably result from the use of a transformation somewhat different from that used in the control condition. On the other hand, this demonstration of a substantial decrement in performance clearly indicates that subjects could not implement the usual transformation.

In the following, we will entertain altcrnate explanations for the processing that we have postulated. We will consider the various stages of the sensorimotor transformation from the representation of the target position to the representation of the intended movement. We will also compare our postulated parcellation in the calculation of arm orientations to the parcellation that has been described for orienting movements of the body, head, and eyes.

\section{Representation of target parameters}

We have assumed that subjects ignored particular target parameters and used a reduced set of parameters in the transformation to arm orientations. An alternate possibility is that subjects began each trial by mentally altering the target parameters. Subjects could have performed a mental rotation of the target into the sagittal plane when they matched target elevation and into 
the horizontal plane when they matched target azimuth (Georgopoulos et al., 1989). The $100 \%$ increase in variable error when subjects matched elevation ("ignoring" distance and azimuth) could possibly be due to variability added by this mental rotation. However, this alternate hypothesis predicts a similar additional variability in the task of matching azimuth ("ignoring" distance and elevation), since this task would involve an analogous mental rotation of target direction. This prediction was not satisfied by our experimental results.

\section{Channel separation}

In the experimental conditions in which subjects matched elevation and distance or matched azimuth, there was a small decrement in performance. Variable error increased slightly and accuracy (as measured by the slope of the regression coefficient) decreased slightly. One possible interpretation is that the parcellation into 2 separate channels is not complete. However, other factors may also contribute to this increase in error. First, all the experimental conditions except the control condition presented the subjects with novel task demands and we gave them very little practice. Second, the transformation from target location to arm angles is but 1 step in a series of transformations that ultimately leads to patterned muscle activity (Soechting and Flanders, 1990). It is possible that the different experimental conditions also affect these other steps. For example, there are no arm muscles that produce only yaw movements. Therefore, the pattern of muscle activity in all the arm muscles most likely differed under various experimental conditions.

\section{Parcellation of intended movements}

A parcellation of a different sensorimotor transformation into an azimuthal component and a component related to distance and elevation has been suggested by Grobstein (1988) and his colleagues. They investigated the body movements of frogs in response to prey presented in different parts of space. This response involves the optic tectum of the frog since lesions of parts of the tectum can abolish the responses to prey in a particular region in space. Brain-stem lesions caudal to the tectum can selectively abolish a channel dealing only with the transformation of information about azimuth. Rather than failing to respond to eccentric stimuli, this brain-stem-lesioned frog responds as if these stimuli were located at zero azimuth (Kostyk and Grobstein, 1987; Masino and Grobstein, 1989). The response does not simply lack the yaw (or azimuthal) motor component, since the relationship of the movement to target distance and elevation is that normally seen for targets at zero azimuth, and not for eccentric targets. Therefore, this lesion affects the transformation of azimuth information prior to the computation of a yaw motor component.

More recently, Masino and Knudsen (1989) have provided similar evidence of a parcellation into horizontal (azimuthal) and vertical (elevation) components of the head movements in barn owls. Stimulation of the birds' tectum leads to a head movement which depends on the locus of the stimulus. The system is refractory to a second stimulus presented after a short interval. By means of stimulation of 2 different sites in short succession, they showed a refractoriness to only the horizontal or the vertical component of the head movement, depending on which component was elicited by the first stimulus. These results may be analogous to those obtained by Robinson (1972) for eye movement saccades elicited by electrical stimulation of the superior colliculus (see also, Tomlinson and Bahra, 1986).
However, the extraocular muscles of the eye pull horizontally or vertically, whereas neck muscles pull in oblique directions (Keshncr et al., 1989). Therefore, while the results for cyc movements are ambiguous with regard to this issue, it is clear that the parcellation observed for head movements occurs prior to the specification of motor commands.

Like the motor cortex (Georgopoulos, 1986; Georgopoulos et al., 1988), the primate superior colliculus (and presumably the tectum of other vertebrates) encodes a representation of "motor error," or the movement needed to get from the current position to the intended final position (Mays and Sparks, 1980; Sparks, 1988; Waitzman et al., 1988). In our schematic diagram of successive processing stages (Fig. 1) this corresponds to the Intended Movement computed from the initial position and the target position. If the tectum of frogs and birds encodes the direction of the intended movement rather than just the target position, this would suggest that the parcellation reported in previous studies occurs at a later stage of processing than the parccllation wc are now reporting for the computation of arm orientations. Like head movements, arm movements are implemented by muscles that pull in many oblique directions. The transformation from intended movement to the production of motor commands is beyond the scope of Figure 1. How this transformation is accomplished and whether or not it involves a second parcellation are open questions.

\section{References}

Georgopoulos AP (1986) On reaching. Annu Rev Neurosci 9:147170.

Georgopoulos AP, Kettner RE, Schwartz AB (1988) Primate motor cortex and free arm movements to visual targets in three-dimensional space. II. Coding the direction by a neuronal population. J Neurosci $8: 2928-2937$.

Georgopoulos AP, Lurito JT, Petrides M, Schwartz AB, Massey JT (1989) Mental rotation of the neuronal population vector. Science 243:234-236.

Grobstein P (1988) Between the retinotectal projection and directed movement: topography of a sensorimotor interface. Brain Behav Evol 31:34-48.

Keshner EA, Campbell D, Katz RT, Peterson BW (1989) Neck muscle activation patterns in humans during isometric head stabilization. Exp Brain Res 75:335-344.

Kostyk SK, Grobstein P (1987) Neuronal organization underlying visually elicited prey orienting in the frog. I. Effects of various unilateral lesions. Neuroscience 21:41-55.

Masino T, Grobstein P (1989) The organization of descending tectofugal pathways underlying orienting in the frog, Rana pipiens. I. Lateralization, parcellation, and an intermediate representation. Exp Brian Res 75:227-244.

Masino T, Knudsen EI (1990) Horizontal and vertical components of head movements are controlled by distinct neural circuits in the barn owl. Nature (in press).

Mays LE, Sparks DL (1980) Saccades are spatially, not retinocentrically, coded. Science 208:1163-1165.

Robinson DA (1972) Eye movements evoked by collicular stimulation in the alert monkey. Vision Res 12:1795-1808.

Soechting JF, Flanders M (1989a) Sensorimotor representations for pointing to targets in three-dimensional space. J Neurophysiol 62 $582-594$

Soechting JF, Flanders M (1989b) Errors in pointing are due to approximations in sensorimotor transformations. J Neurophysiol 62: 595-608.

Soechting JF, Flanders M (1990) Deducing central algorithms of arm movement control from kinematics. Dahlem konferenzen proceedings (in press).

Soechting JF, Ross B (1984) Psychophysical determination of coordinate representation of human arm orientation. Neuroscience 13: 595-604.

Soechting JF, Tillery SIH, Flanders, M (1990) Transformation from 
head- to shoulder-centercd representation of target direction in arm movements. J Cogn Neurosci 2:32-43.

Sparks, DL (1988) Saccadic command signals in the superior colliculus: implications for sensorimotor transformations. Can J Physiol Pharmacol 66:527-531.

Tomlinson RD, Bahra PS (1986) Combined eye-head gaze shifts in the primate. II. Intcractions betwecn saccades and the vestibuloocular reflex. J Neurophysiol 56:1558-1570.

Waitzman DM, Ma TP, Optican LM, Wurtz RH (1988) Superior colliculus neurons provide the saccadic motor error signal. Exp Brain Res 72:649-652. 